\title{
GRB 190114C in the nuclear region of an interacting galaxy
}

\section{A detailed host analysis using ALMA, the HST, and the VLT ${ }^{\star}$}

\author{
A. de Ugarte Postigo ${ }^{1,2}$, C. C. Thöne ${ }^{1}$, S. Martín 3,4 , J. Japelj ${ }^{5}$, A. J. Levan ${ }^{6,7}$, M. J. Michałowski ${ }^{8}$, J. Selsing ${ }^{9,10}$, \\ D. A. Kann ${ }^{1}$, S. Schulze ${ }^{11}$, J. T. Palmerio ${ }^{12,13}$, S. D. Vergani ${ }^{12}$, N. R. Tanvir ${ }^{14}$, K. Bensch ${ }^{1}$, S. Covino ${ }^{15}$, \\ V. D’Elia ${ }^{16,17}$, M. De Pasquale ${ }^{18}$, A. S. Fruchter ${ }^{19}$, J. P. U. Fynbo ${ }^{9,10}$, D. Hartmann ${ }^{20}$, K. E. Heintz ${ }^{21}$, \\ A. J. van der Horst ${ }^{22,23}$, L. Izzo ${ }^{1,2}$, P. Jakobsson ${ }^{21}$, K. C. Y. Ng ${ }^{12,24}$, D. A. Perley ${ }^{25}$, A. Rossi ${ }^{26}$, B. Sbarufatti ${ }^{27}$, \\ R. Salvaterra ${ }^{28}$, R. Sánchez-Ramírez ${ }^{29}$, D. Watson ${ }^{9,10}$, and D. $\mathrm{Xu}^{30}$
}

(Affiliations can be found after the references)

Received 10 September 2019 / Accepted 18 November 2019

\begin{abstract}
Context. For the first time, very high energy emission up to the TeV range has been reported for a gamma-ray burst (GRB). It is still unclear whether the environmental properties of GRB 190114C might have contributed to the production of these very high energy photons, or if it is solely related to the released GRB emission.

Aims. The relatively low redshift of the GRB $(z=0.425)$ allows us to study the host galaxy of this event in detail, and to potentially identify idiosyncrasies that could point to progenitor characteristics or environmental properties that might be responsible for this unique event.

Methods. We used ultraviolet, optical, infrared, and submillimetre imaging and spectroscopy obtained with the HST, the VLT, and ALMA to obtain an extensive dataset on which the analysis of the host galaxy is based.

Results. The host system is composed of a close pair of interacting galaxies $\left(\Delta v=50 \mathrm{~km} \mathrm{~s}^{-1}\right)$, both of which are well detected by ALMA in $\mathrm{CO}(3-2)$. The GRB occurred within the nuclear region ( $170 \mathrm{pc}$ from the centre) of the less massive but more star-forming galaxy of the pair. The host is more massive $\left(\log \left(M / M_{\odot}\right)=9.3\right)$ than average GRB hosts at this redshift, and the location of the GRB is rather unique. The higher star formation rate was probably triggered by tidal interactions between the two galaxies. Our ALMA observations indicate that both host galaxy and companion have a high molecular gas fraction, as has been observed before in interacting galaxy pairs.

Conclusions. The location of the GRB within the core of an interacting galaxy with an extinguished line of sight is indicative of a denser environment than typically observed for GRBs and could have been crucial for the generation of the very high energy photons that were observed.
\end{abstract}

Key words. gamma-ray burst: individual: 190114C - ISM: molecules - galaxies: ISM - galaxies: star formation

\section{Introduction}

Gamma-ray burst (GRB) host-galaxy studies have been important for constraining the properties and nature of the progenitor objects (Hjorth et al. 2012; Vergani et al. 2015; Krühler et al. 2015; Perley et al. 2016a,b; Palmerio et al. 2019). Different types of progenitors are expected to be found in different types of galaxies, or in particular regions within them (Fruchter et al. 2006; Kelly et al. 2008, 2014; Svensson et al. 2010; Modjaz et al. 2011; Sanders et al. 2012; Lyman et al. 2017; Japelj et al. 2018). Most of the studies performed on GRB hosts have been based on observations in the optical and nearinfrared range, where they have traditionally been more feasible. Until recently, observations of the host galaxies of GRBs at longer wavelengths were limited to small samples that were necessarily biased towards the very brightest objects because of the

* Partially based on Hubble Space Telescope observations obtained under Director's Discretionary Time programme number 15684 (P.I.: Levan) and under programme number 15708 (P.I.: Levan). Partially based on Very Large Telescope observations obtained by the Stargate Consortium under programme 0102.D-0662 (P.I.: Tanvir). Partially based on Atacama Large Millimeter Array observations obtained under Director's Discretionary Time programme ADS/JAO.ALMA\#2018.A.00020.T (P.I.: de Ugarte Postigo). sensitivity of the observatories (Tanvir et al. 2004; Hatsukade et al. 2011; Michałowski et al. 2012; de Ugarte Postigo et al. 2012), and by the limited availability of space observatories (Hunt et al. 2014; Schady et al. 2014). In particular, one of the most relevant ranges of study lies at millimetre and submillimetre wavelengths, where emission of cold dust within galaxies can be observed. This wavelength range also covers many of the interesting atomic and molecular features associated with the clouds where GRB progenitors are expected to form. Furthermore, light emitted in this wavelength range is not affected by dust obscuration, enabling us to penetrate the densest regions of the galaxies. In the past years, new powerful millimetre and submillimetre observations, such as those performed by the Atacama Large Millimeter/submillimeter Array (ALMA) or the NOrthern Extended Millimeter Array (NOEMA), are allowing us to study GRBs and their galaxies in detail using photometric (Wang et al. 2012; Hatsukade et al. 2014; Sánchez-Ramírez et al. 2017; Laskar et al. 2018), spectroscopic (Michałowski et al. 2016; de Ugarte Postigo et al. 2018; Hatsukade et al. 2019), and polarimetric (Laskar et al. 2019) techniques.

GRB 190114C, localised (Gropp et al. 2019) by the Neil Gehrels Swift Observatory (Swift hereafter; Gehrels et al. 2004), is the first GRB with confirmed very high energy (VHE) emission (up to one $\mathrm{TeV}$ ), and is thus a milestone in high-energy astrophysics (Mirzoyan et al. 2019). The GRB had one of the 

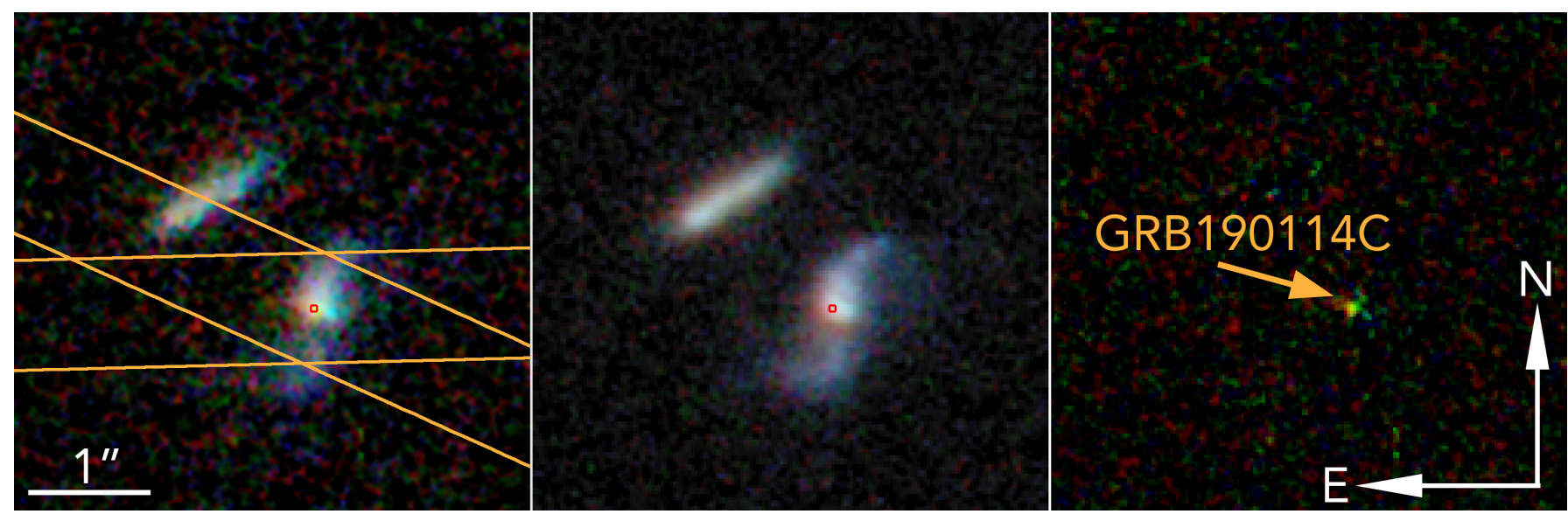

Fig. 1. Colour composites obtained from the combination of the $F 606 \mathrm{~W}, F 775 \mathrm{~W}$, and $F 850 L P$ HST observing bands. The left image is made from the 11 February data, and the central image from the 28 August data. Right panel: subtraction of both frames, showing that the optical counterpart was still well detected in February. The location of the GRB is indicated with a red circle. The orange lines in the left panel indicate the position of the slits during the two X-shooter observations.

highest peak photon fluxes detected by BAT (Krimm et al. 2019), and was found to be at a redshift of $z=0.425$ (Selsing et al. 2019; Castro-Tirado et al. 2019). The sightline was significantly extinguished (Kann et al. 2019). Despite this, the early afterglow was exceedingly bright (Lipunov et al. 2019). Archival imaging from the Panoramic Survey Telescope And Rapid Response System (Pan-STARRS) revealed what was later confirmed to be an underlying host galaxy (de Ugarte Postigo et al. 2019).

Although HE $\gamma$-rays, as have been observed by the Fermi Large Area Telescope (Fermi-LAT), can be caused by the high-energy tail of bright afterglow emission (see Ajello et al. 2019 for an overview of observations and their interpretation), no VHE photons have been observed to exceed $\approx 160 \mathrm{GeV}$ (rest-frame; see Takahashi 2019 for some very recent results). It has been proposed that the production of VHE emission could be due to other processes involved in the interaction of the burst ejecta with the circumburst medium (see e.g. Böttcher \& Dermer 1998; Pe'er \& Waxman 2005; Fan et al. 2008; Wang et al. 2010; for reviews: Inoue et al. 2013; Nava 2018). If a particularly dense environment were related to the VHE emission, we would expect to find VHE-emitting events in a particular region of the galaxy where such conditions would be more favourable, such as the core of the galaxy or locations with a large amount of line-of-sight absorption ${ }^{1}$.

In this paper we present optical, near-infrared, and submillimetre photometry and spectroscopy of the host galaxy of GRB 190114C. We explore the peculiarities of this event and any distinctive factor that could have favoured the generation of the VHE photons that were observed.

Section 2 presents the observational data, and Sect. 3 the results of our imaging and spectroscopic analyses. In Sect. 4 we discuss the implications of our findings. Section 5 presents our conclusions. Throughout the paper we use a cosmology with $H_{0}=71 \mathrm{~km} \mathrm{~s}^{-1} \mathrm{Mpc}^{-1}, \Omega_{\mathrm{M}}=0.27$, and $\Omega_{\Lambda}=0.73$ (Spergel et al. 2003). Unless specifically stated, the errors provided within this paper are $1 \sigma$.

\footnotetext{
1 The recently discovered very nearby GRB 190829A (The Fermi GBM team 2019; Dichiara et al. 2019), which has also been detected at extremely high energies (de Naurois 2019), also lies behind a highly extinguished line of sight (Heintz et al. 2019, J. Bolmer, priv. comm.).
}

\section{Observations}

\subsection{Optical and near-infrared imaging from the HST with ACS and WFPC3}

The Hubble Space Telescope (HST) observed the field of GRB 190114C using the Advanced Camera for Surveys (ACS, Sirianni et al. 2005) at three epochs, within the context of the Director's Discretionary Time (DDT) programme number 15684 (P.I.: Levan), searching for the optical counterpart. The field was observed in four optical bands: $F 475 W, F 606 W, F 775 W$, and $F 850 L P$. Observations in all bands were obtained on 11 February 2019 and in $F 475 W$ and $F 850 L P$ also on 12 March 2019 (the additional bands were not obtained at this epoch due to a guide-star acquisition failure). A visit with the missing filters $F 606 W$ and $F 775 W$ was obtained on 28 June 2019. To complete the dataset, a final visit was obtained on 28 August 2019 through the regular time programme number 15708 (P.I.: Levan). We obtained imaging in $F 606 W, F 775 W$, and $F 850 L P$ optical filters with ACS, and $F 110 W, F 160 W$ infrared filters with the Wide Field Camera 3 (WFC3, MacKenty et al. 2010). Data were first corrected for charge transfer efficiency (CTE) corrections to remove significant CTE features due to the harsh radiation environment, as well as the removal of bias striping. Processed images were then reduced and combined via astrodrizzle with a final pixel scale of 0 '.025/pixel. Images were co-aligned by the use of point sources in common to the different filters.

The GRB is located near the core of its host galaxy, at $00^{\prime \prime} 030 \pm 0$ ' 010 (or a projected distance of $170 \pm 60 \mathrm{pc}$ ) towards the southeast of the galaxy's light centre. This is shown by image subtraction in the $F 775 \mathrm{~W}$ and $F 850 L P$ bands, where both afterglow and host are well detected, with consistent offsets in both bands.

The host galaxy has a projected size of $1^{\prime \prime} .6 \times 0 \times 0^{\prime \prime} 8$, equivalent to $9.1 \times 4.5 \mathrm{kpc}$, as measured from the $3 \sigma$ detection contours of an image combining all the visible bands. We also measure a radius of the galaxy of $r_{50}=0, ! 34=1.90 \mathrm{kpc}$ (containing $50 \%$ of the light), or $r_{80}=00^{\prime \prime} 62=3.45 \mathrm{kpc}$ (containing $80 \%$ of the light). These radii were determined using the method described by Blanchard et al. (2016).

In addition to the host galaxy of the GRB, another galaxy is detected at a distance of 1 '!2 $2 \pm 0$ "'1 (see Fig. 1). In this paper we confirm based on the ALMA spectroscopy described in Sect. 2.5 
Table 1. Magnitudes (AB system) for the host galaxy, not corrected for Galactic extinction.

\begin{tabular}{lcccc}
\hline \hline Filter & Source & $\begin{array}{c}T-T_{0} 0 \\
\text { (day) }\end{array}$ & Host mag & Comp. mag \\
\hline FUV & GALEX & pre & \multicolumn{2}{c}{$24.60 \pm 0.32$} \\
NUV & GALEX & pre & \multicolumn{2}{c}{$24.12 \pm 0.15$} \\
\hline F475W & HST/ACS & 221 & $23.10 \pm 0.28$ & $24.34 \pm 0.31$ \\
F606W & HST/ACS & 221 & $22.31 \pm 0.16$ & $22.95 \pm 0.21$ \\
F775W & HST/ACS & 221 & $21.88 \pm 0.19$ & $22.21 \pm 0.22$ \\
F850LP & HST/ACS & 221 & $21.60 \pm 0.24$ & $21.86 \pm 0.27$ \\
F110W & HST/WFC3 & 221 & $21.24 \pm 0.10$ & $21.46 \pm 0.11$ \\
$J$ & VLT/HAWK-I & 63 & $21.27 \pm 0.07$ & $21.18 \pm 0.07$ \\
F160W & HST/WFC3 & 221 & $21.10 \pm 0.10$ & $21.26 \pm 0.11$ \\
$H$ & VLT/HAWK-I & 63 & $20.83 \pm 0.11$ & $20.95 \pm 0.10$ \\
$K_{S}$ & VLT/HAWK-I & 63 & $20.69 \pm 0.20$ & $20.61 \pm 0.20$ \\
\hline $3.4 \mu \mathrm{m}$ & WISE & pre & $20.11 \pm 0.11$ \\
$4.6 \mu \mathrm{m}$ & WISE & pre & $20.65 \pm 0.40$ \\
\hline
\end{tabular}

Notes. $T-T_{0}$ indicates the epoch of the observation in days since the burst onset, where pre indicates data obtained in a pre-explosion epoch.

that both galaxies lie at the same redshift. At a redshift of $z=$ 0.425 , this is equivalent to a projected distance of $6.8 \pm 0.6 \mathrm{kpc}$.

The counterpart is detected in the reddest bands during the first epoch (Acciari et al. 2019), being particularly prominent in the $F 775 \mathrm{~W}$ band. In this paper we use the data from the fourth epoch, in which the GRB contribution is negligible, to study the host galaxy. The photometry was performed with SExtractor (Bertin \& Arnouts 1996) using elliptical apertures with sizes based on the Kron radius (Kron 1980), and using the zero-points provided by the Space Telescope Science Institute for each of the filters at the specific epoch of the observation (Sirianni et al. 2005). The values obtained using this method (see Table 1) were compared to other aperture photometry methods, yielding similar results.

\subsection{Near-infrared imaging from VLT/HAWK-I}

More than two months after the GRB, on 23 March 2019 the field was observed in the near-infrared (NIR) bands with HAWK-I (High-Acuity Wide-field $K$-band Imager; Pirard et al. 2004) mounted on the fourth Unit Telescope (UT4) of the Very Large Telescope (VLT), managed by the European Southern Observatory (ESO). The observation was performed within the Stargate collaboration programme 0102.D-0662 (P.I.: Tanvir). Both galaxies are well detected and resolved in the three observing bands $J, H$, and $K_{S}$, with a seeing of $00^{\prime \prime} 60,00^{\prime \prime} 55$, and $0^{\prime \prime} 60$, respectively. This allowed us to perform independent photometry of the host and the companion galaxy. Data reduction was performed with a dedicated pipeline based on IRAF routines. We obtained seeing-matched aperture photometry calibrated with respect to field stars from the 2MASS catalogue (Skrutskie et al. 2006). This photometry is shown in Table 1.

\subsection{Ultraviolet and infrared archival photometry from GALEX and WISE}

We complement the host-galaxy photometry with catalogue ultraviolet observations from GALEX (Bianchi et al. 2011) and infrared observations from WISE (Wright et al. 2010). Unfortunately, the spatial resolutions of GALEX $\left(4^{\prime \prime 2} 2\right.$ in the farultraviolet, FUV, and 5'.3 in the near-ultraviolet, NUV, band) and of WISE (6".1 in the $3.4 \mu \mathrm{m}$ and $6 . .4$ in the $4.6 \mu \mathrm{m}$ band) are not enough to resolve the two objects, and we can only obtain combined photometry of both galaxies.

The GALEX photometry was obtained from the GALEX Merged Catalog (MCAT) GR6/7 Data Release ${ }^{2}$. For WISE observations we used the photometry from the AllWISE source catalogue $^{3}$, transforming them from Vega to $\mathrm{AB}$ system with the corresponding AB-to-Vega offsets of 2.699 and 3.339 mag in the 3.4 and $4.6 \mu \mathrm{m}$ bands, respectively (Tokunaga \& Vacca 2005).

\subsection{VLT/X-shooter spectroscopy}

Spectroscopy of GRB 190114C was obtained with the three-arm X-shooter echelle spectrograph (Vernet et al. 2011), mounted on UT2 of VLT (programme 0102.D-0662, P.I.: Tanvir). Two epochs of X-shooter observations were obtained, beginning $4.8 \mathrm{~h}$ and $76 \mathrm{~h}$, respectively, after the Swift trigger (see Thöne et al., in prep. for more details). The initial results were reported by Kann et al. (2019). The first observation was carried out using the $11^{\prime \prime} 0 / 0 \prime 99 / 0 \prime 99$ slit, with observations lasting $4 \times 600 \mathrm{~s}$. The second observation consisted of an $8 \times 1200$ s spectroscopic integration with the $11^{\prime \prime} 0 / 0 \prime \prime 9 / 0 \prime \prime 9 \mathrm{JH}$ slit. The observations were performed using a nodding scheme with a 5',0 nodding throw. Because the host is small and the surrounding regions are mostly empty, this allows for a very clean background subtraction. In this paper we use the second observation to perform emission-line analysis of the host galaxy of GRB 190114C. This second epoch was divided into two executions of a $4 \times 1200 \mathrm{~s}$ observing block. The slit was aligned with the parallactic angle at the beginning of each execution (see Fig. 1), and all the data were then combined to produce the spectrum that we used in this paper. The combined observation included the core of the host galaxy in the slit, with no significant contribution from the companion galaxy. The resulting spectrum covers the spectral range between 3200 and $18000 \AA$.

\subsection{ALMA CO(3-2) imaging and spectroscopy}

Millimetre observations, tuned to cover the $\mathrm{CO}(3-2)$ line at the redshift of the GRB (i.e. $242.663 \mathrm{GHz}$ ), were carried out with the ALMA observatory in Band 6, within the context of DDT programme ADS/JAO.ALMA\#2018.A.00020.T (P.I.: de Ugarte Postigo). Five individual executions were performed in three independent epochs ranging between 17 and 18 January 2019. The configuration used 47-48 antennas with baselines ranging from $15 \mathrm{~m}$ to $313 \mathrm{~m}(12-253 \mathrm{k} \lambda$ at the observed frequency). Each observation consisted of 43 min integration time on source with average weather conditions of precipitable water vapour $\sim 3-4 \mathrm{~mm}$. The correlator was configured for a central observed frequency of $235.0487 \mathrm{GHz}$. The data were calibrated within CASA (Common Astronomy Software Applications, version 5.4.0, McMullin et al. 2007) using the pipeline calibration. The spatial resolution of the spectral data cube obtained by the pipeline products that combined all five executions was $1^{\prime \prime} .16 \times 00^{\prime \prime} 867$ (position angle $-87.9^{\circ}$ ), which was just enough to resolve the host galaxy and companion and perform independent analysis. The spectroscopic measurements were also analysed within CASA. The flux calibration was performed using the quasar J0522-3627 in the first two epochs and with J0423-0120 for the last one.

The $\mathrm{CO}(3-2)$ zero-moment image is shown in Fig. 2. To image the $\mathrm{CO}$ emission, continuum subtraction was performed

\footnotetext{
http://galex.stsci.edu/GR6/

https://irsa.ipac.caltech.edu/Missions/wise.html
} 


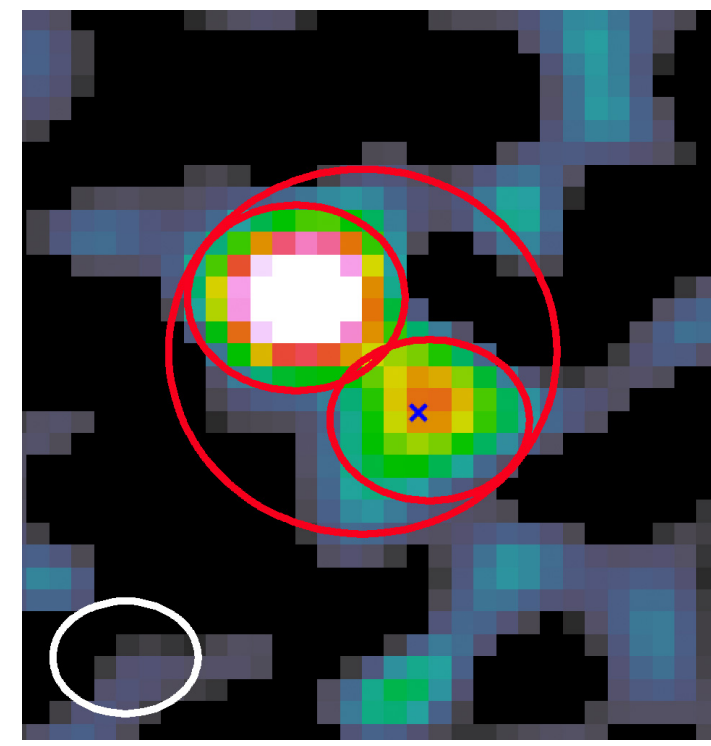

Fig. 2. Image of the $\mathrm{CO}(3-2)$ emission obtained by ALMA. The spatial resolution (indicated by the white ellipse in the lower left corner) is just enough to resolve the two interacting galaxies. The red elliptical regions indicate the apertures we used to measure the fluxes shown in Table 3. The blue cross indicates the location of the afterglow.

in the UV visibilities using line-free channels on both sides of $\mathrm{CO}(3-2)$. The spectral ranges used to fit the continuum were $241.839-242.433$ and $242.800-243.574 \mathrm{GHz}$, which corresponds to a line-emitting region of $\sim 450 \mathrm{~km} \mathrm{~s}^{-1}$. In order to ensure that the $\mathrm{CO}$ map in Fig. 2 was not contaminated by residual continuum, we integrated several random velocity ranges within the considered line free regions. No significant residual continuum was detected in those verification maps.

\section{Results}

\subsection{SED fits}

In Table 1 we show the observed magnitudes of the host galaxy of GRB 190114C and its companion, based on the datasets described in Sect. 2. These observations were corrected for Galactic extinction $(E(B-V)=0.011$, Schlafly \& Finkbeiner 2011) before we proceeded with the spectral energy distribution (SED) fitting.

We used LePhare (Arnouts et al. 1999; Ilbert et al. 2006) to fit these photometric data with a set of galaxy templates based on the models of Bruzual \& Charlot (2003) and a Calzetti extinction law (Calzetti et al. 2000). LePhare uses an exponentially declining star formation history (SFH) and a Chabrier initial mass function (IMF, Chabrier 2003).

To be able to include the unresolved photometry provided by GALEX and WISE, we used the following iterative process: First we obtained an SED fit using only the resolved photometry from the HST and VLT. Using these models, we estimated the fraction with which each of the galaxies would be contributing to the photometry in each of the unresolved bands, and used this fraction to estimate the photometry of each of the galaxies in the above-mentioned bands based on the blended photometry. Then, the SED fit was performed again using all the bands. The process was iterated until it converged. This process required only a few iterations, rapidly converging to the parameters shown in Table 2. Figure 3 shows the different fits. Although the GALEX and WISE photometry helps to better constrain the galaxy
Table 2. Properties of the host galaxy and companion derived from the SED analysis.

\begin{tabular}{lcc}
\hline \hline Property & Host & Companion \\
\hline$\chi^{2} / \#$ filters & $9.1 / 13$ & $14.6 / 13$ \\
$E(B-V)(\mathrm{mag})$ & 0.30 & 0.10 \\
Age $(\mathrm{Gyr})$ & $0.23_{-0.18}^{+1.30}$ & $6.12_{-3.55}^{+2.40}$ \\
$\log \left(\operatorname{Mass}\left(M_{\odot}\right)\right)$ & $9.27_{-0.25}^{+0.28}$ & $9.95_{-0.22}^{+0.09}$ \\
SFR $\left(M_{\odot} \mathrm{yr}^{-1}\right)$ & $9.4_{-6.4}^{+12.4}$ & $0.28_{-0.15}^{+0.51}$ \\
$\log \left(\operatorname{SSFR}\left(\mathrm{yr}^{-1}\right)\right)$ & $-8.3_{-0.8}^{+0.6}$ & $-10.5_{-0.4}^{+0.6}$ \\
\hline
\end{tabular}

models, the results differ only slightly from those obtained with the HST/ACS and VLT/HAWK-I data alone.

\subsection{Molecular analysis}

The submillimetre data at the time of our observation were dominated by the afterglow emission (for a detailed analysis of the continuum emission, see Acciari et al. 2019; Misra et al. 2019). This implies that we cannot study the continuum emission of the host galaxy.

After subtracting the afterglow continuum, we are left with the $\mathrm{CO}(3-2)$ emission, which reveals the two galaxies identified by the HST, for which we obtained independent spectroscopy. Using the apertures shown in Fig. 2, we extracted the spectra of the GRB host, the companion galaxy, and the combined system. The $\mathrm{CO}(3-2)$ lines detected in all of these spectra were used to calculate the flux density, redshift, full width at half-maximum (FWHM), and relative velocity of the galaxies. From the $\mathrm{CO}$ flux, and knowing the distance to the galaxy, we can determine the luminosity of the line, and with some assumptions, determine the expected star formation rate (SFR) for the amount of $\mathrm{CO}$ and the expected mass of $\mathrm{H}_{2}$. We used two assumptions for the conversion factor of $\mathrm{CO}$ to $\mathrm{H}_{2}$. First we used $\alpha_{\mathrm{CO}}=5 M_{\odot} /\left(\mathrm{K} \mathrm{km} \mathrm{s}^{-1} \mathrm{pc}^{2}\right)$ (we drop the unit in what follows) in order to enable direct comparison with previous work. Then, we used the metallicity-dependent calibration of Amorín et al. (2016), which for the metallicity of the host results in $\alpha_{\mathrm{CO}}=18.2$. This spectral analysis is presented in Table 3. As was already evident in Fig. 2, the companion galaxy is more luminous in the $\mathrm{CO}(3-2)$ transition than the host, whereas in the optical emission the host galaxy of GRB 190114C dominates.

The $\mathrm{CO}$ emission of both galaxies is almost coincident in redshift. The centres of the emission lines differ by only $50 \pm$ $20 \mathrm{~km} \mathrm{~s}^{-1}$ (see Fig. 4). This confirms that the two galaxies are, indeed, at the same distance. Together with the small projected distance between the two galaxies and the somewhat disturbed morphology shown by the GRB host, this suggests that this is an interacting system.

The centre of the host galaxy and the afterglow are located close to each other, but they are not completely coincident. Using the ALMA data, we measure a distance between them of $0.094 \pm 0$ '.065, which is equivalent to a projected distance of $530 \pm 370 \mathrm{pc}$. This is larger than the $0 \prime \prime 030 \pm 0 \prime \prime 010$ or a projected distance of $170 \pm 60 \mathrm{kpc}$ derived from the HST imaging, but consistent within the errors, resulting from the larger uncertainty of the ALMA astrometry. In any case, a displacement of the molecular gas centroid with respect to the optical galaxy core could be a consequence of the galaxy interaction that we are observing. 


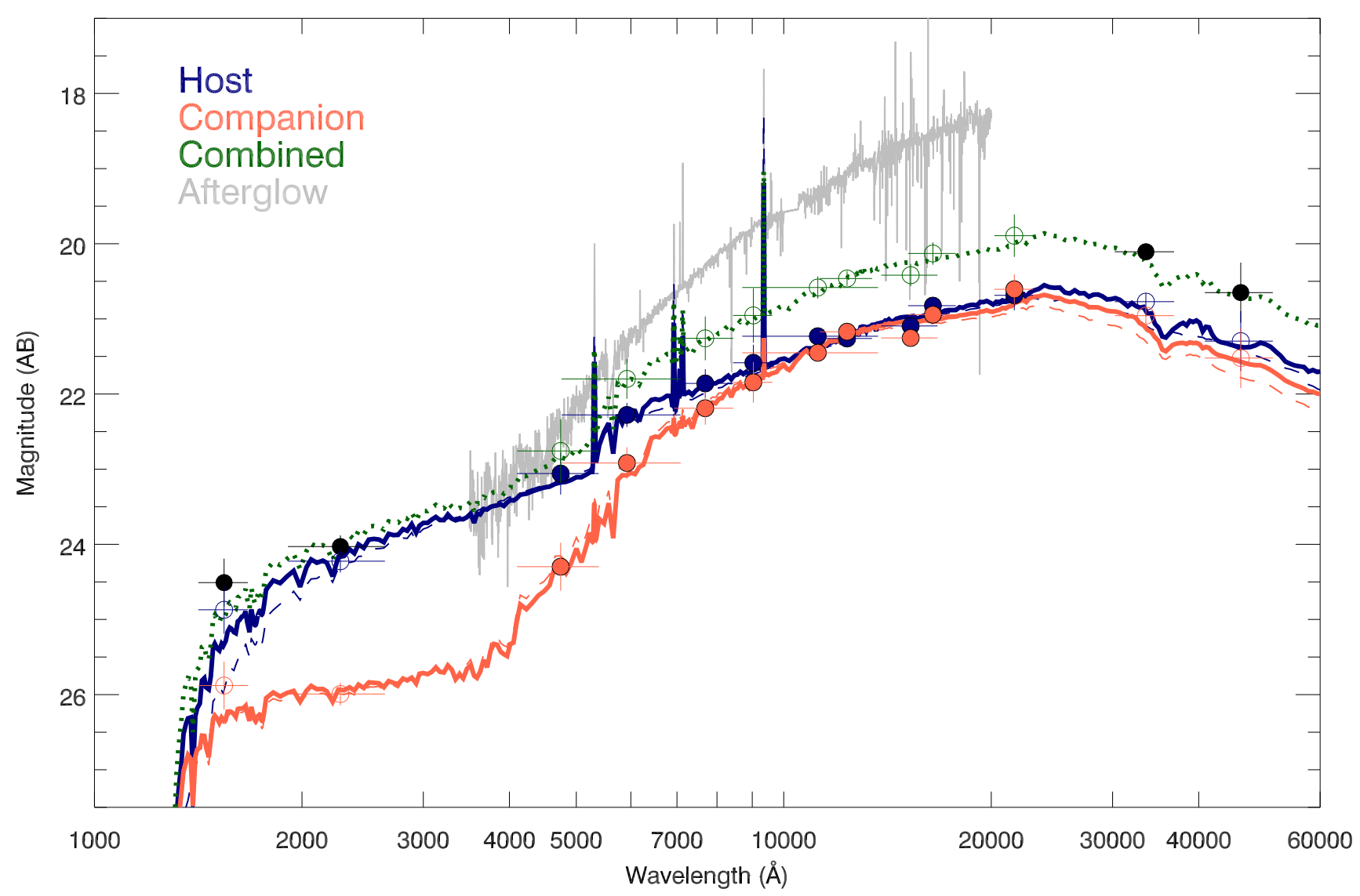

Fig. 3. SED of the host galaxy of GRB $190114 \mathrm{C}$ (blue) and its companion (red). The dashed lines indicate the fits without considering the GALEX and WISE photometry, where the two objects are blended. The solid lines are the fits considering this photometry as well. In green we show the combined light of both galaxies. The spectrum of the second visit of X-shooter, which is afterglow-dominated above $5000 \AA$, is plotted in grey.

Table 3. Spectral analysis from the ALMA CO(3-2) spectroscopy.

\begin{tabular}{lccc}
\hline \hline Property & Host & Companion & Combined \\
\hline Amplitude $(\mathrm{mJy})$ & $0.61 \pm 0.10$ & $1.37 \pm 0.10$ & $2.16 \pm 0.15$ \\
Centre $(\mathrm{GHz})$ & $242.625 \pm 0.010$ & $242.654 \pm 0.005$ & $242.646 \pm 0.004$ \\
$F W H M\left(\mathrm{~km} \mathrm{~s}^{-1}\right)$ & $232 \pm 42$ & $231 \pm 19$ & $223 \pm 19$ \\
Line flux $\left(\mathrm{mJy} \mathrm{km} \mathrm{s}^{-1}\right)$ & $150 \pm 25$ & $336 \pm 26$ & $512 \pm 41$ \\
\hline$L_{\mathrm{CO}(3-2)}\left(L_{\odot}\right)$ & $(2.04 \pm 0.34) \times 10^{5}$ & $(4.57 \pm 0.35) \times 10^{5}$ & $(6.97 \pm 0.56) \times 10^{5}$ \\
$L_{\mathrm{CO}(3-2)}^{\prime}\left(\mathrm{K} \mathrm{km} \mathrm{s}^{-1} \mathrm{pc}^{2}\right)$ & $(1.54 \pm 0.26) \times 10^{8}$ & $(3.45 \pm 0.27) \times 10^{8}$ & $(5.26 \pm 0.42) \times 10^{8}$ \\
$L_{\mathrm{CO}(1-0)}^{\prime}\left(\mathrm{K} \mathrm{km} \mathrm{s}^{-1} \mathrm{pc}^{2}\right)^{(a)}$ & $(2.75 \pm 0.46) \times 10^{8}$ & $(6.17 \pm 0.48) \times 10^{8}$ & $(9.40 \pm 0.75) \times 10^{8}$ \\
\hline $\mathrm{SFR}(\mathrm{CO})\left(M_{\odot} \mathrm{yr}^{-1}\right)^{(b)}$ & $33 \pm 8$ & $74 \pm 15$ & $113 \pm 21$ \\
$M_{\mathrm{H}_{2}}\left(M_{\odot}\right)^{(c)}\left[\alpha_{\mathrm{CO}}=5\right]$ & $(1.38 \pm 0.23) \times 10^{9}$ & $(3.08 \pm 0.24) \times 10^{9}$ & $(4.70 \pm 0.38) \times 10^{9}$ \\
$f_{\mathrm{gas}}(d)\left[\alpha_{\mathrm{CO}}=5\right]$ & $0.7_{-0.4}^{+0.7}$ & $0.35_{-0.12}^{+0.26}$ & $0.44_{-0.14}^{+0.29}$ \\
$M_{\mathrm{H}_{2}}\left(M_{\odot}\right)^{(e)}\left[\alpha_{\mathrm{CO}}=18.2\right]$ & $(5.0 \pm 0.8) \times 10^{9}$ & $(11.2 \pm 0.9) \times 10^{9}$ & $(17.1 \pm 1.4) \times 10^{9}$ \\
$f_{\mathrm{gas}}(d)\left[\alpha_{\mathrm{CO}}=18.2\right]$ & $2.7_{-1.6}^{+2.5}$ & $1.3_{-0.4}^{+1.0}$ & $1.6_{-0.5}^{+1.0}$ \\
\hline
\end{tabular}

Notes. ${ }^{(a)}$ Assuming $L_{\mathrm{CO}(3-2)}^{\prime} / L_{\mathrm{CO}(1-0)}^{\prime}=0.56$ (Carilli \& Walter 2013) for star-forming galaxies. ${ }^{(b)}$ Based on the relation given by Hunt et al. (2015) for metal-poor galaxies. ${ }^{(c)}$ Assuming a Galactic CO-to- $\mathrm{H}_{2}$ conversion factor of $\alpha_{\mathrm{CO}}=5 M_{\odot} /\left(\mathrm{K} \mathrm{km} \mathrm{s}^{-1} \mathrm{pc}^{2}\right)$. ${ }^{(d)} \mathrm{Calculated}_{\text {as }} f_{\mathrm{gas}}=M_{\mathrm{H}_{2}} / M_{*}$. ${ }^{(e)}$ Assuming a metallicity-dependent CO-to- $\mathrm{H}_{2}$ conversion factor (Amorín et al. 2016) of $\alpha_{\mathrm{CO}}=18.2 M_{\odot} /\left(\mathrm{K} \mathrm{km} \mathrm{s}^{-1} \mathrm{pc}^{2}\right.$ ).

\subsection{Host emission-line analysis}

We detect a number of emission lines from the host in the second epoch X-shooter spectrum (see Table 4). These include the usual prominent strong lines, but we also detect [S III] $\lambda$ 9071 and emission lines of [ $\mathrm{Ne}$ III] and He I $\lambda 5876$ and 10833. The latter are only present in young stellar populations of age $<50 \mathrm{Myr}$ (González Delgado et al. 1999).
The absolute flux calibration of the spectrum was finetuned using the simultaneous Gamma-ray Burst Optical/ Near-infrared Detector (GROND) multiband photometric measurements (Acciari et al. 2019). Several lines are affected by telluric absorption, and especially the Balmer lines show signs of absorption along the line of sight in the host. To measure the fluxes of these lines, we masked the contaminated 

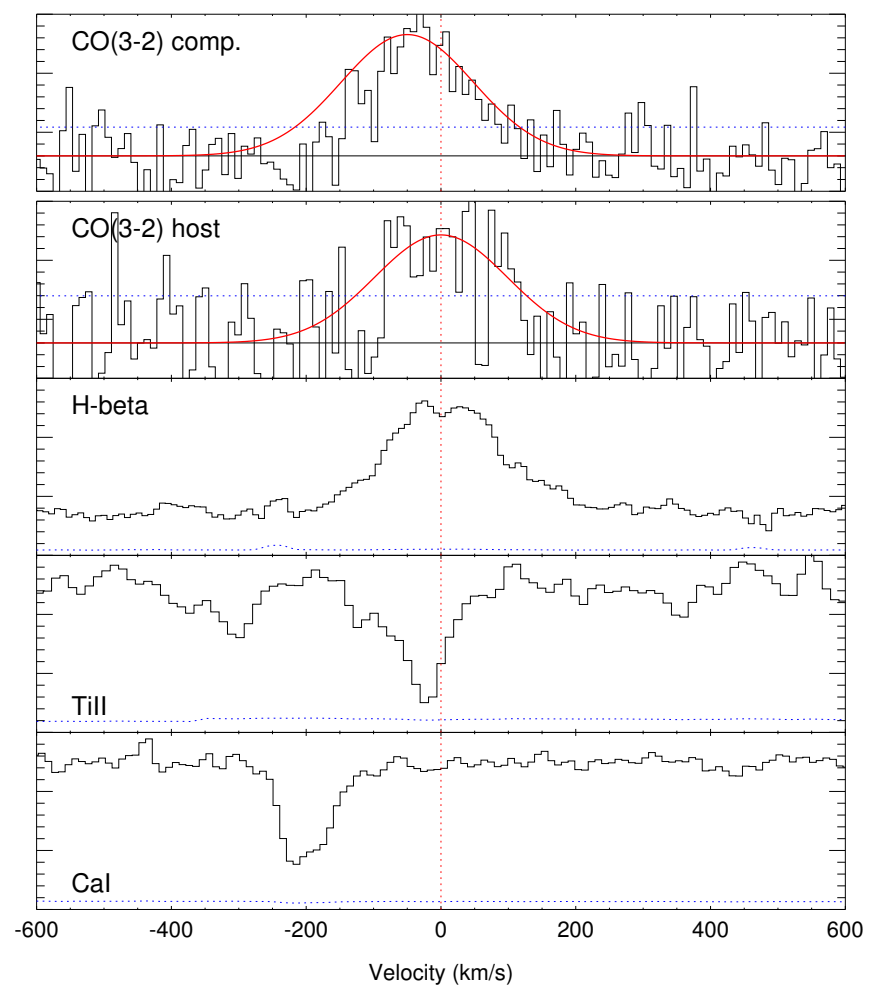

Fig. 4. Comparison of the lines detected in the ALMA and X-shooter spectra. This plot shows the coincidence of the host emission lines in both the ALMA and X-shooter spectra. However, the absorption features that are present in the spectrum are displaced and cover a much wider range in velocities than the emission, even when compared with the interacting companion galaxy, which is shown in the top panel.

regions and fitted the remaining part of the emission line with a Gaussian function. Fluxes of non-contaminated lines were measured by simple integration. Errors were obtained through a Monte Carlo (MC) simulation using the error spectrum and were cross-checked with the rms of the spectral continuum. For 1000 simulated events we repeatedly added random Gaussian noise (standard deviations were taken from the error spectra) to the best-fit model and fitted the resulting spectrum by the same model. The obtained distribution of best-fit parameters was then used to compute the $1-\sigma$ errors. In addition, the $\mathrm{H} \beta$ and $\mathrm{H} \gamma$ lines are likely affected by underlying stellar Balmer absorption, as suggested by the high stellar mass. Because even the secondepoch X-shooter spectrum is dominated by afterglow emission, however, we cannot directly search for the wings of the absorption around the Balmer lines.

To determine the host-averaged attenuation, we compared the Balmer decrement to the theoretical predictions for an electron temperature of $10^{4} \mathrm{~K}$ and densities of $10^{2}-10^{4} \mathrm{~cm}^{-2}$ (i.e. case B recombination; Osterbrock 1989). The attenuation was derived by simultaneously minimising the ratios of the $\mathrm{H} \alpha, \mathrm{H} \beta$, and $\mathrm{H} \gamma$ lines and assuming different extinction curves: the average extinction curves of the Milky Way (MW, Pei 1992) and the Small Magellanic Cloud (SMC, Pei 1992), as well as the attenuation curve for starburst galaxies (Calzetti et al. 2000). Errors were obtained through an MC simulation. The three different curves give slightly different results, but all point towards a relatively high value. For the MW extinction we obtain $E(B-V)=$ $0.83 \pm 0.03 \mathrm{mag}$, for the SMC $E(B-V)=0.76 \pm 0.02 \mathrm{mag}$, and a starburst attenuation curve gives $E(B-V)=0.92 \pm 0.03$ mag. Our analysis does not show any strong preference for a particular type of attenuation.
Table 4. Emission lines, identified in the X-shooter spectrum, and their measured fluxes corrected for Galactic extinction only.

\begin{tabular}{|c|c|}
\hline Line & $\begin{array}{c}F_{\lambda} \\
{\left[10^{-17} \mathrm{erg} \mathrm{cm}^{-2} \mathrm{~s}^{-1}\right]}\end{array}$ \\
\hline [O II] 3726 & $12.6 \pm 0.3$ \\
\hline [O II $] 3729$ & $16.2 \pm 0.3$ \\
\hline$[\mathrm{Ne} \text { III }]^{(a)}$ & $2.9 \pm 0.4$ \\
\hline $\mathrm{H} \gamma^{(b)}$ & $4.3 \pm 0.3$ \\
\hline $\mathrm{H} \beta^{(b)}$ & $14.5 \pm 0.3$ \\
\hline [O III $] 4959^{(a)}$ & $12.8 \pm 0.3$ \\
\hline [O III $] 5007^{(a)}$ & $38.8 \pm 0.3$ \\
\hline He I 5876 & $2.9 \pm 0.5$ \\
\hline [O I] 6300 & $2.3 \pm 0.3$ \\
\hline $\mathrm{H} \alpha$ & $89.7 \pm 0.8$ \\
\hline [N II $] 6583$ & $14.8 \pm 0.6$ \\
\hline [S II] 6717 & $8.9 \pm 0.3$ \\
\hline [S II] 6733 & $7.1 \pm 0.3$ \\
\hline [S III] $9071^{(a)}$ & $10.9 \pm 0.5$ \\
\hline He I 10833 & $11.2 \pm 0.5$ \\
\hline $\operatorname{Pa} \gamma^{(c)}$ & $4.9 \pm 0.5$ \\
\hline
\end{tabular}

Notes. We did not correct for host extinction here because of the uncertainty in deriving the extinction (see text). ${ }^{(a)}$ Fluxes obtained by simple integration, ${ }^{(b)}$ The line is affected by line-of-sight absorption in the afterglow continuum, fluxes are obtained by fitting a single Gaussian to the emission part. ${ }^{(c)}$ The line suffers from residual sky emission, the flux is obtained by using the unaffected blue wing of the line and approximating it with a Gaussian with a fixed centre.

The attenuation-corrected $\mathrm{H} \alpha$ flux was then used to estimate the SFR, adopting the Kennicutt (1998) relation for a Chabrier (2003) IMF. The values range from SFR 13-25 $M_{\odot} \mathrm{yr}^{-1}$, depending on the extinction relation used. The electron density was derived from the ratio of the [O II] lines: $n_{\mathrm{e}}=87 \pm 0.16 \mathrm{~cm}^{-3}$ (Osterbrock 1989). A similar value is obtained through the ratio of the [S II] $\lambda \lambda 6727,6732$ doublet. Finally, we also determined metallicities using two calibrators based on the recalibration of the $\mathrm{O} 3 \mathrm{~N} 2$ and $\mathrm{N} 2$ parameters with $T_{\mathrm{e}}$-obtained metallicities in Marino et al. (2013). For O3N2 we obtain $12+\log (\mathrm{O} / \mathrm{H})=8.27 \pm$ 0.03 , while for $\mathrm{N} 2$ we derive $12+\log (\mathrm{O} / \mathrm{H})=8.38 \pm 0.02$. The more reliable metallicity determination from the electron temperature cannot be applied here because the $T_{\mathrm{e}}$-sensitive [O III] $\lambda 4363$ line is not detected. We note that the $\mathrm{O} 3 \mathrm{~N} 2$ parameter might suffer slightly from unaccounted stellar absorption in $\mathrm{H} \beta$, but the values from both calibrators are consistent within errors.

\section{Discussion}

The host of GRB $190114 \mathrm{C}$ has a projected size of 1 ".6 6 0 ${ }^{\prime \prime} 8$, and a half-light radius of $r_{50}=1.90 \mathrm{kpc}$ (or an $80 \%$ light radius of $r_{80}=3.45 \mathrm{kpc}$ ). We compare this with the samples of Lyman et al. (2017) and Blanchard et al. (2016), who used HST data of 39 and 105 GRB hosts, respectively. The Lyman et al. (2017) sample comprises only observations with the F160W near-infrared filter, but Blanchard et al. (2016) used whatever filter was available for each of the hosts in the HST archive, with preference towards optical data from the ACS. Lyman et al. (2017) determined an average radius of $r_{50}=1.7 \pm 0.2 \mathrm{kpc}$ $\left(r_{80}=3.1 \pm 0.4 \mathrm{kpc}\right)$, which is similar to the value obtained by Blanchard et al. (2016) of $r_{50}=1.8 \pm 0.1 \mathrm{kpc}\left(r_{80} \sim 3 \mathrm{kpc}\right)$. This means that the host galaxy of our study is slightly larger than average, but well within what is typically found for GRB hosts. 


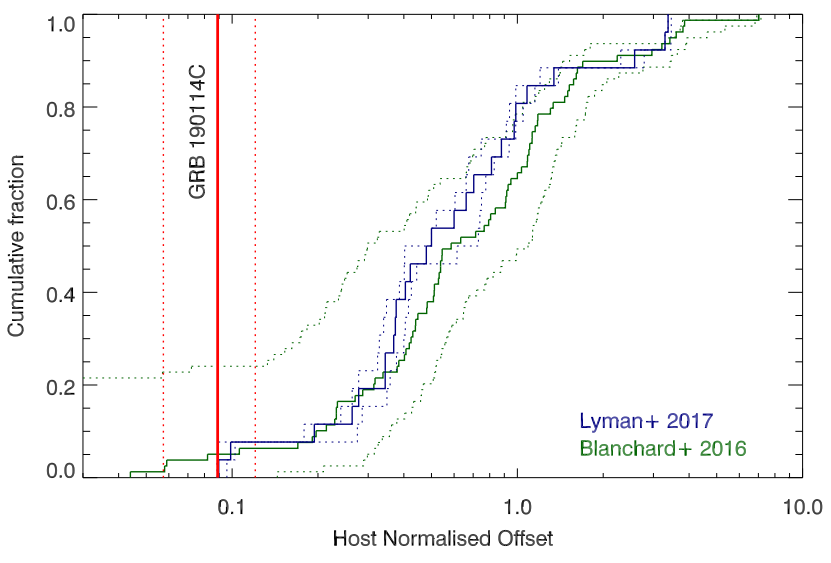

Fig. 5. Cumulative distribution of host normalised offsets for the samples of Blanchard et al. (2016) and Lyman et al. (2017). The dotted lines indicate the 1- $\sigma$ uncertainties. The host normalised offset of GRB 190114C is shown in red, and lies at the lower end of the distribution.

Our galaxy shows a tidally disrupted morphology, with the GRB occurring within the bulge of the galaxy at $170 \pm 60 \mathrm{pc}$ from the galaxy centre. This offset is significantly smaller than the average found for GRBs, which was measured to be $1.0 \pm 0.2 \mathrm{kpc}$ by Lyman et al. (2017) or $1.3 \pm 0.2 \mathrm{kpc}$ by Blanchard et al. (2016). By examining the location of the host galaxy in a BPT diagram (Baldwin et al. 1981), we can discard the presence of a central active galactic nucleus (AGN) and any additional variability produced by it. This allows us to conclude that the detected emission is only related to the GRB, and the offset measured from the host galaxy core is accurate. The location of GRB $190114 \mathrm{C}$ is even more outstanding when we consider the normalised offset, calculated as the ratio between the measured offset and the half-light radius of the galaxy (see Fig. 5). The average values measured within the HST GRB host samples were Offset $/ r_{50}=0.6 \pm 0.1$ (Lyman et al. 2017) and Offset $/ r_{50}=0.7 \pm 0.2$. In our case, we measure Offset $/ r_{50}=0.09 \pm 0.03$.

A second galaxy is located at the same redshift, with a velocity difference of only $50 \pm 20 \mathrm{~km} \mathrm{~s}^{-1}$ and a projected distance of $6.8 \pm 0.6 \mathrm{kpc}$, suggesting that the two galaxies interact. The mass ratio between the two galaxies is $M_{\text {comp }} / M_{\text {host }}=4.8_{-3.5}^{+1.9}$, implying that the interacting system can be classified as a minor merger, which are those with a mass ratio higher than 3.0 (Scudder et al. 2012), although the uncertainty is large and could make the system also consistent with a major merger. Furthermore, the system can be classified as a close pair, which includes galaxies with a projected separation $r_{\mathrm{p}}<30 \mathrm{kpc}$. Interacting systems result in a higher SFR, which is even higher in the case of major mergers and is maximised in the case of close pairs At a distance of less than $10 \mathrm{kpc}$, Scudder et al. (2012) showed that major mergers have an average increase in SFR of a factor of two, whereas minor mergers have increases of a factor in the range 1.5-2. The interaction also results in a decrease in the metallicity of the system, as compared to non-interacting galaxies. Although this shows a weaker trend as the projected distance changes, it is also maximised for the closest interacting galaxies, where the metallicity changes by $\Delta \log (\mathrm{O} / \mathrm{H}) \sim-0.05$.

Violino et al. (2018) found in a study of molecular gas in galaxy pairs that they have shorter depletion times and an enhancement of the molecular gas fraction of 0.4 dex than a control sample of isolated galaxies, although this is consistent with other galaxies with similarly enhanced SFR. The host of GRB 190114C is not deficient in molecues, as has been found in other GRB hosts (Michałowski et al. 2018a), and the molecular gas fractions of host and companion are higher than those found in the control sample. We note that Violino et al. (2018) used a CO-to- $\mathrm{H}_{2}$ conversion factor that was lower than any of those that we used, but even considering this, the molecular gas fractions of the GRB host and the companion galaxy are consistent with the galaxy-pair sample and larger than that of the control sample.

The increase in SFR and the decrease in metallicity found in galaxy pairs are factors that favour the production of the stars that end up generating a long GRB on timescales of a few dozen Myr. Together with the high molecular-gas fraction found in the GRB host and companion, we therefore suggest that the influence of the interaction on the current conditions of the system was a key factor in generating the conditions that gave rise to the progenitor of GRB 190114C.

The host is star forming, as shown in the different methods that we applied in our analysis. The SED fit reveals a somewhat unconstrained value of SFR $=9.4_{-6.4}^{+12.8} M_{\odot} \mathrm{yr}^{-1}$, whereas the analysis of the strong emission lines implies an SFR that ranges from 13 to $25 M_{\odot} \mathrm{yr}^{-1}$, depending on the extinction law used. Although the values that we obtain vary, they consistently show significant star formation activity within the host. This is not the case for the companion galaxy, for which we estimate an $\mathrm{SFR}=0.28_{-0.15}^{+0.51} M_{\odot} \mathrm{yr}^{-1}$ from the SED fit.

The total SFR and molecular gas masses are known to be correlated. Directly from the CO luminosity, and assuming low metallicity $(12+\log (\mathrm{O} / \mathrm{H})<8.4)$, we can estimate an SFR of $33 \pm 8 M_{\odot} \mathrm{yr}^{-1}$ (Hunt et al. 2015) for the host galaxy, while we obtain an SFR $=74 \pm 15 M_{\odot} \mathrm{yr}^{-1}$ for the companion. However, in the case of the companion, the low-metallicity assumptions are probably not valid (although we do not have a measurement of it), leading to an overestimated SFR from the CO data. In any case, this is clearly a molecule-rich galaxy.

Using a different approach based on the relation derived by Michałowski et al. (2018a, Eq. (1)), for SFR $=9.4-25 M_{\odot} \mathrm{yr}^{-1}$ of the GRB $190114 \mathrm{C}$ host, we can predict $\log M_{\mathrm{H}_{2}}=10.05-10.49$. This is significantly higher than our estimated value $\log M_{\mathrm{H}_{2}}=$ 9.14 with $\alpha_{\mathrm{CO}}=5$ or 9.69 with a metallicity-dependent factor (Amorín et al. 2016) of $\alpha_{\mathrm{CO}}=18.2$, which means taht the host would be molecule deficient, as has been claimed for a few other GRB hosts (Hatsukade et al. 2014; Stanway et al. 2015; Michałowski et al. 2016, 2018a). On the other hand, the companion galaxy with SFR $=0.28 M_{\odot} \mathrm{yr}^{-1}$ is expected to have $\log M_{\mathrm{H}_{2}}=8.44$, an order of magnitude lower than the measured value. The companion is therefore very molecule rich compared to other galaxies with similar SFR. In the top panel of Fig. 6, adapted from Michałowski et al. (2018a), we plot the two galaxies as compared to other GRB hosts and several galaxy samples (Hunt et al. 2014, 2015, 2017; Cormier et al. 2014; Grossi et al. 2016; Young et al. 1989; Leroy et al. 2008; Solomon et al. 1997; Sanders et al. 1991; Bothwell et al. 2013, 2014; Bertemes et al. 2018; Krumholz et al. 2011; Daddi et al. 2010). However, the CO-to$\mathrm{H}_{2}$ conversion factor depends on metallicity (Bolatto et al. 2013). This was parametrised by Michałowski et al. (2018a, Eq. (3)) as a relation between molecular-gas depletion time $M_{\mathrm{H}_{2}} / \mathrm{SFR}$ and metallicity. For $12+\log (\mathrm{O} / \mathrm{H})=8.3$ for the GRB $190114 \mathrm{C}$ host, this relation predicts $\log M_{\mathrm{H}_{2}}=9.21-9.64$, consistent with the value that we find (see the bottom panel of Fig. 6).

The resulting gas-to-stellar ratios of the host and the companion of 0.74 and 0.44 , respectively, are within the values found for other galaxies (Leroy et al. 2008; Boselli et al. 2010, 2014; Bothwell et al. 2014; Michałowski et al. 2015). The value of 2.7 derived for the host using $\alpha_{\mathrm{CO}}=18.2$ is close to the highest measurements for other galaxies. 

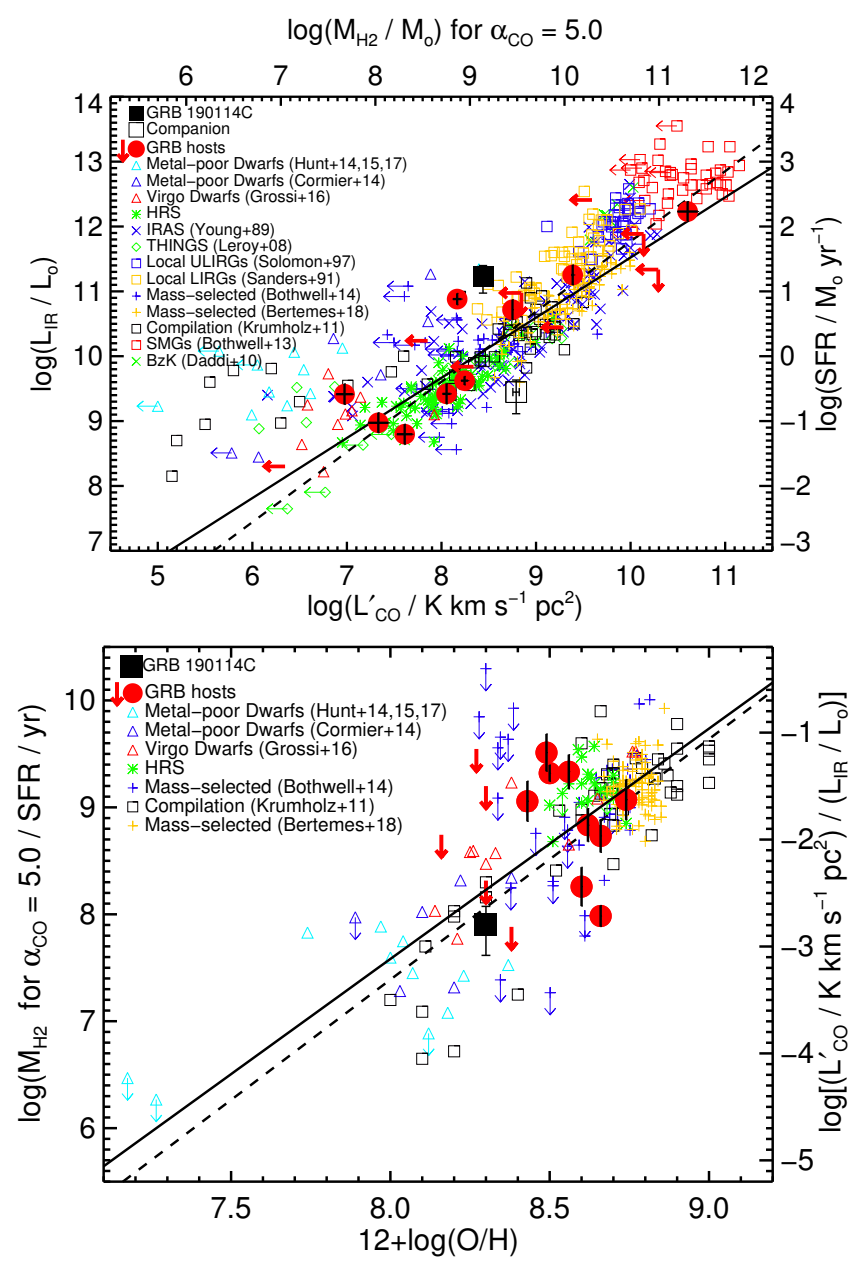

Fig. 6. Top: infrared luminosity and SFR as a function of the $\mathrm{CO}$ luminosity, using a CO-to- $\mathrm{H}_{2}$ conversion factor of $\alpha_{\mathrm{CO}}=$ $5 M_{\odot} /\left(\mathrm{K} \mathrm{km} \mathrm{s}^{-1} \mathrm{pc}^{2}\right)$. The host of GRB $190114 \mathrm{C}$ is indicated with a black filled square, and the companion is shown with an empty square. They are compared to the existing sample of GRB host galaxies and other galaxy samples. Bottom: molecular gas depletion time (or the inverse of the star-formation efficiency), i.e. the ratio of the CO luminosity to the infrared luminosity or the corresponding molecular gas mass with the CO-to- $\mathrm{H}_{2}$. In this case, only the host galaxy is shown because the metallicity is not available for the companion, together with several galaxy samples. Adapted from Michałowski et al. (2018a).

To place the emission-line properties of the host in the context of other long GRB hosts, we compared the mass-metallicity (MZ) relation and the SFR/SSFR-mass relation to those of other long GRBs as well as to samples from the literature up to redshifts of $\sim 2$. In Fig. 7 we plot the MZ relation of long GRB hosts compared to galaxy samples and the fit to the MZ relation at different redshifts. In contrast to many other comparisons in the literature, we derive metallicities consistently from one single calibrator (with one exception for the VIMOS-VLT Deep Survey, VVDS, extreme emission-line galaxies, EELG, sample): the N2 parameter in the Marino et al. (2013) calibration. The host of GRB 190114C falls slightly below the MZ relations at $z=0.07$ and even $z=0.7$, but has a relatively high mass compared to other GRB hosts at this redshift. The stellar mass is $\sim 1$ dex higher than the median value of GRB hosts at $0<z<1$ as measured for the BAT6 host sample (Vergani et al. 2015), but it is consistent with those of $z>1$ hosts (Palmerio et al. 2019). However, GRB 190114C was close to the centre of a large spiral galaxy, which usually has a nega-

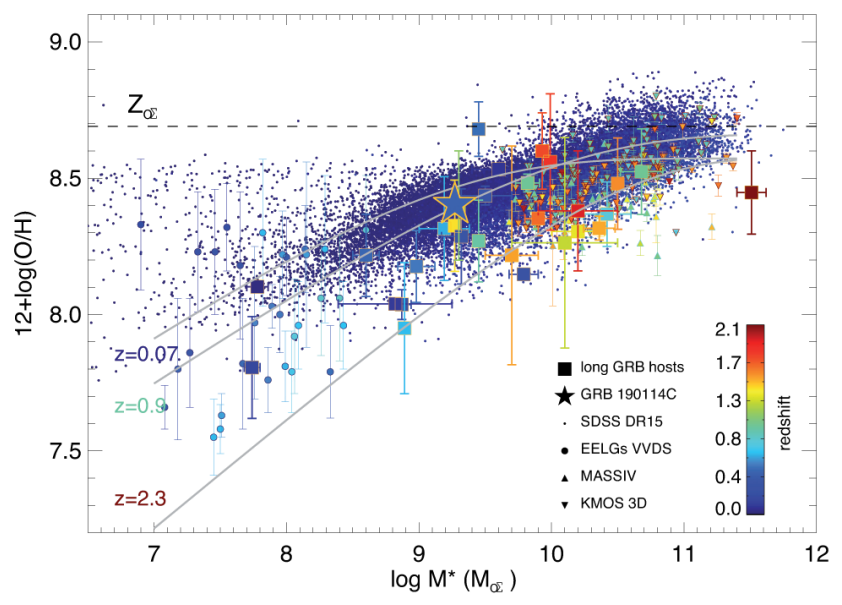

Fig. 7. Mass-metallicity relation for the host of GRB $190114 \mathrm{C}$ and other long GRB hosts. As comparison samples we plot data from SDSS DR15 $(z=0-0.32)(\mathrm{https}: / /$ www . sdss.org/dr15/), the KMOS3D sample $(z=0.6-2.7$, Wuyts et al. 2016), extreme emission-line galaxies found in the VVDS ( $z=0-0.9$, Amorín et al. 2014) and the MASSIVE sample $(z=0.9-2.2$, galaxies from the VVDS sample followed up by SINFONI, Contini et al. 2012) (http: //cosmosdb.iasf-milano. inaf.it:8080/VVDS-SINFONI/). We only plot galaxies up to a redshift of 2.1 for better visualisation. All metallicities except those in the Amorín et al. (2014) EELG sample are derived from the N2 parameter and the Marino et al. (2013) calibration. Amorín et al. (2014) metallicities were derived with the $T_{\mathrm{e}}$ method. We also plot the $\mathrm{MZ}$ relations at $z=0.07$ (Zahid et al. 2013), $z=0.9$ and $z=2.3$ (Wuyts et al. 2014), which are also based on N2 parameter metallicities. The MZ relations were corrected for the difference in metallicity calibrators.

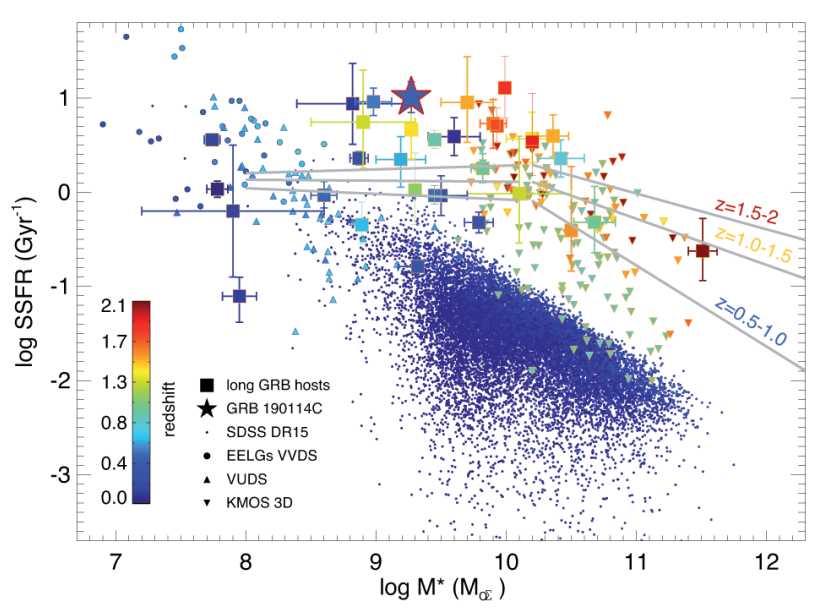

Fig. 8. Specific SFR vs. stellar mass relation for the host of GRB 190114C and other long GRB hosts. The specific SFR was obtained by weighting it with the stellar mass. References to the comparison samples are the same as in Fig. 7, we also add a sample of star-forming galaxies from the VUDS survey (Calabrò et al. 2017), but leave out the MASSIVE sample. SFRs for the comparison samples were largely derived by SED fitting, except for the KMOS3D sample. The grey lines are fits with a broken power law and a cut-off mass of log $M=10.2 M_{\odot}$ at different redshifts derived by Whitaker et al. (2014). Error bars for the comparison samples are omitted for clarity.

tive metallicity gradient (decreasing towards the outskirts of the galaxy), while in other spiral GRB hosts, the GRB occurred in the outer spiral arms (see e.g. Izzo et al. 2019). The GRB site is therefore likely more metal rich than the measurements that we can make of the overall galaxy from strong-line analysis and could be close to solar, which would be exceptional for a 
GRB site (but see e.g. Elliott et al. 2013; Schady et al. 2015; Michałowski et al. 2018b).

We also compare the SFR and SSFR (SFR weighted by the stellar mass) with other GRB hosts and field galaxies. The SSFRmass relation is plotted in Fig. 8. The correlation between SFR and mass for star-forming galaxies is a broken power law with a mass cut-off of $\log M \sim 10.2 M_{\odot}$ or possibly somewhat higher at $z>1$ (Whitaker et al. 2014). Translated into the SSFR, this implies a constant SSFR below the mass cut-off, while the relation drops at higher masses. Long GRB hosts have on average higher SSFRs than field emission-line galaxies from the SDSS (Japelj et al. 2016; Palmerio et al. 2019), for example, and SSFR-M relations derived from other star-forming galaxy samples (see e.g. Whitaker et al. 2014). The SSFR of the host of GRB 190114C also lies above the relation for typical starforming galaxies and its SSFR is on the upper end of the distribution of long GRBs at $z<0.5$. The metallicity, SFR, SSFR, and stellar mass of the host galaxy of GRB 190114C do not show any striking peculiarities with respect to other GRB hosts, except for the relatively high stellar mass for its redshift, a slightly higher SSFR, and the atypical location of the GRB in the nucleus of the galaxy.

\section{Conclusions}

More detailed studies of the close local environment of the GRB are required to determine how peculiar this event was and whether the potentially dense environment could have produced the observed VHE photons. Our main conclusions are listed below:

1. Imaging with high spatial resolution from the HST showed that the host-galaxy system of GRB 190114C is composed of two galaxies at a projected separation of $6.8 \pm 0.6 \mathrm{kpc}$.

2. ALMA $\mathrm{CO}(3-2)$ spectroscopy confirmed that both galaxies are located at the same redshift, with a very low radial velocity difference. This is indicative that they interact in a plane that is almost perpendicular to the line of sight.

3. These two galaxies constitute a close-pair merger system. These systems are known to result in a higher SFR and a decrease in metallicity, which favour the production of GRB progenitors.

4. Both host galaxy and companion have a large molecular gas fraction, probably induced by the interaction. The fraction is larger than typically found in isolated galaxies.

5. The host galaxy of GRB $190114 \mathrm{C}$ has a stellar mass of $\log \left(M / M_{\odot}\right)=9.27_{-0.28}^{+0.25}$, which is higher than typical GRB hosts at this redshift. The metallicity is $12+\log (\mathrm{O} / \mathrm{H})=8.3$ or $43 \%$ solar, which is lower than typical field galaxies with similar mass.

6. The host galaxy is star forming. The SFR has been measured using different methods, with values in the range $3-25 M_{\odot} \mathrm{yr}^{-1}$.

7. The He I emission lines in the spectrum indicate a young stellar population, in spite of the greater (although unconstrained) age derived from the SED fit.

8. The GRB exploded within the central cluster of the host galaxy, where the density is higher, at a projected distance of $\sim 170 \mathrm{pc}$ from the core. This is at the lower end of the offsets found for long GRBs, and is even more extreme when we normalise the offset with the size of the host galaxy.

9. The fact that the GRB occurred in an extinguished line of sight, within the nuclear region of a galaxy that is more massive than the average GRB host at this redshift, and that has a significant molecular gas fraction is indicative of a par- ticularly dense environment, which could have been crucial for generating the VHE photons observed by MAGIC.

Acknowledgements. This paper makes use of the following ALMA data: ADS/JAO.ALMA\#2018.A.00020.T. ALMA is a partnership of ESO (representing its member states), NSF (USA) and NINS (Japan), together with NRC (Canada), MOST and ASIAA (Taiwan), and KASI (Republic of Korea), in cooperation with the Republic of Chile. The Joint ALMA Observatory is operated by ESO, AUI/NRAO and NAOJ. CT, AdUP, DAK, MB, and LI acknowledge support from the Spanish research project AYA2017-89384-P. CT and AdUP acknowledge support from funding associated to Ramón y Cajal fellowships (RyC-2012-09984 and RyC-2012-09975). DAK acknowledges support from the Spanish research project RTI2018-098104-J-I00. M.J.M. acknowledges the support of the National Science Centre, Poland through the SONATA BIS grant 2018/30/E/ST9/00208. JPUF thanks the Carlsberg Foundation for support. KEH and PJ acknowledge support by a Project Grant (162948-051) from The Icelandic Research Fund. JS and DW are supported in part by Independent Research Fund Denmark grant DFF-7014-00017. The Cosmic Dawn Center is supported by the Danish National Research Foundation under grant No. 140.

\section{References}

Acciari, V. A., Ansoldi, S., Antonelli, L. A., et al. 2019, Nature, 575, 459 Ajello, M., Arimoto, M., Axelsson, M., et al. 2019, ApJ, 878, 52

Amorín, R., Sommariva, V., Castellano, M., et al. 2014, A\&A, 568, L8 Amorín, R., Muñoz-Tuñón, C., Aguerri, J. A. L., \& Planesas, P. 2016, A\&A, 588, A23

Arnouts, S., Cristiani, S., Moscardini, L., et al. 1999, MNRAS, 310, 540 Baldwin, J. A., Phillips, M. M., \& Terlevich, R. 1981, PASP, 93, 5 Bertemes, C., Wuyts, S., Lutz, D., et al. 2018, MNRAS, 478, 1442 Bertin, E., \& Arnouts, S. 1996, A\&AS, 117, 393

Bianchi, L., Herald, J., Efremova, B., et al. 2011, Ap\&SS, 335, 161

Blanchard, P. K., Berger, E., \& Fong, W.-F. 2016, ApJ, 817, 144 Bolatto, A. D., Wolfire, M., \& Leroy, A. K. 2013, ARA\&A, 51, 207 Boselli, A., Eales, S., Cortese, L., et al. 2010, PASP, 122, 261

Boselli, A., Cortese, L., \& Boquien, M. 2014, A\&A, 564, A65 Bothwell, M. S., Smail, I., Chapman, S. C., et al. 2013, MNRAS, 429, 3047 Bothwell, M. S., Wagg, J., Cicone, C., et al. 2014, MNRAS, 445, 2599 Böttcher, M., \& Dermer, C. D. 1998, ApJ, 499, L131 Bruzual, G., \& Charlot, S. 2003, MNRAS, 344, 1000

Calabrò, A., Amorín, R., Fontana, A., et al. 2017, A\&A, 601, A95 Calzetti, D., Armus, L., Bohlin, R. C., et al. 2000, ApJ, 533, 682 Carilli, C. L., \& Walter, F. 2013, ARA\&A, 51, 105

Castro-Tirado, A. J., Hu, Y., Fernandez-Garcia, E., et al. 2019, GRB Coordinates Network, 23708

Chabrier, G. 2003, PASP, 115, 763

Contini, T., Garilli, B., Le Fèvre, O., et al. 2012, A\&A, 539, A91 Cormier, D., Madden, S. C., Lebouteiller, V., et al. 2014, A\&A, 564, A121

Daddi, E., Bournaud, F., Walter, F., et al. 2010, ApJ, 713, 686

de Naurois, M. 2019, GRB Coordinates Network, 25566, 1 de Ugarte Postigo, A., Lundgren, A., Martín, S., et al. 2012, A\&A, 538, A44 de Ugarte Postigo, A., Thöne, C. C., Bolmer, J., et al. 2018, A\&A, 620, A119 de Ugarte Postigo, A., Kann, D. A., Thoene, C. C., \& Izzo, L. 2019, GRB Coordinates Network, 23692, 1

Dichiara, S., Bernardini, M. G., Burrows, D. N., et al. 2019, GRB Coordinates Network, 25552, 1

Elliott, J., Krühler, T., Greiner, J., et al. 2013, A\&A, 556, A23

Fan, Y.-Z., Piran, T., Narayan, R., \& Wei, D.-M. 2008, MNRAS, 384, 1483

Fruchter, A. S., Levan, A. J., Strolger, L., et al. 2006, Nature, 441, 463

Gehrels, N., Chincarini, G., Giommi, P., et al. 2004, ApJ, 611, 1005

González Delgado, R. M., Leitherer, C., \& Heckman, T. M. 1999, ApJS, 125, 489

Gropp, J. D., Kennea, J. A., Klingler, N. J., et al. 2019, GRB Coordinates Network, 23688, 1

Grossi, M., Corbelli, E., Bizzocchi, L., et al. 2016, A\&A, 590, A27

Hatsukade, B., Kohno, K., Endo, A., Nakanishi, K., \& Ohta, K. 2011, ApJ, 738, 33

Hatsukade, B., Ohta, K., Endo, A., et al. 2014, Nature, 510, 247

Hatsukade, B., Hashimoto, T., Kohno, K., et al. 2019, ApJ, 876, 91

Heintz, K. E., Fynbo, J. P. U., Jakobsson, P., et al. 2019, GRB Coordinates Network, 25563, 1

Hjorth, J., Malesani, D., Jakobsson, P., et al. 2012, ApJ, 756, 187 Hunt, L. K., Palazzi, E., Michałowski, M. J., et al. 2014, A\&A, 565, A112 Hunt, L. K., García-Burillo, S., Casasola, V., et al. 2015, A\&A, 583, A114 Hunt, L. K., Weiß, A., Henkel, C., et al. 2017, A\&A, 606, A99 Ilbert, O., Arnouts, S., McCracken, H. J., et al. 2006, A\&A, 457, 841 
Inoue, S., Granot, J., O’Brien, P. T., et al. 2013, Astropart. Phys., 43, 252 Izzo, L., de Ugarte Postigo, A., Maeda, K., et al. 2019, Nature, 565, 324 Japelj, J., Vergani, S. D., Salvaterra, R., et al. 2016, A\&A, 590, A129 Japelj, J., Vergani, S. D., Salvaterra, R., et al. 2018, A\&A, 617, A105

Kann, D. A., Thoene, C. C., Selsing, J., et al. 2019, GRB Coordinates Network, 23710, 1

Kelly, P. L., Kirshner, R. P., \& Pahre, M. 2008, ApJ, 687, 1201

Kelly, P. L., Filippenko, A. V., Modjaz, M., \& Kocevski, D. 2014, ApJ, 789, 23

Kennicutt, Jr., R. C. 1998, ARA\&A, 36, 189

Krimm, H. A., Barthelmy, S. D., Cummings, J. R., et al. 2019, GRB Coordinates Network, 23724,

Kron, R. G. 1980, ApJS, 43, 305

Krühler, T., Malesani, D., Fynbo, J. P. U., et al. 2015, A\&A, 581, A125

Krumholz, M. R., Leroy, A. K., \& McKee, C. F. 2011, ApJ, 731, 25

Laskar, T., Alexander, K. D., Berger, E., et al. 2018, ApJ, 862, 94

Laskar, T., Alexander, K. D., Gill, R., et al. 2019, ApJ, 878, L26

Leroy, A. K., Walter, F., Brinks, E., et al. 2008, AJ, 136, 2782

Lipunov, V., Tyurina, N., Kuznetsov, A., et al. 2019, GRB Coordinates Network, 23693, 1

Lyman, J. D., Levan, A. J., Tanvir, N. R., et al. 2017, MNRAS, 467, 1795

MacKenty, J. W., Kimble, R. A., O'Connell, R. W., \& Townsend, J. A. 2010, in Society of Photo-Optical Instrumentation Engineers (SPIE) Conference Series, Proc. SPIE, 7731, 77310Z

Marino, R. A., Rosales-Ortega, F. F., Sánchez, S. F., et al. 2013, A\&A, 559, A114

McMullin, J. P., Waters, B., Schiebel, D., Young, W., \& Golap, K. 2007, in Astronomical Data Analysis Software and Systems XVI, eds. R. A. Shaw, F. Hill, \& D. J. Bell, ASP Conf. Ser., 376, 127

Michałowski, M. J., Kamble, A., Hjorth, J., et al. 2012, ApJ, 755, 85

Michałowski, M. J., Gentile, G., Hjorth, J., et al. 2015, A\&A, 582, A78

Michałowski, M. J., Castro Cerón, J. M., Wardlow, J. L., et al. 2016, A\&A, 595, A72

Michałowski, M. J., Karska, A., Rizzo, J. R., et al. 2018a, A\&A, 617, A143

Michałowski, M. J., Xu, D., Stevens, J., et al. 2018b, A\&A, 616, A169

Mirzoyan, R., Noda, K., Moretti, E., et al. 2019, GRB Coordinates Network, 23701,1

Misra, K., Resmi, L., Kann, D. A., et al. 2019, MNRAS, submitted [arXiv:1911.09719]

Modjaz, M., Kewley, L., Bloom, J. S., et al. 2011, ApJ, 731, L4

Nava, L. 2018, Int. J. Mod. Phys. D, 27, 1842003

Osterbrock, D. E. 1989, Astrophysics of Gaseous Nebulae and Active Galactic Nuclei (University Science Books)

Palmerio, J. T., Vergani, S. D., Salvaterra, R., et al. 2019, A\&A, 623, A26

Pe'er, A., \& Waxman, E. 2005, ApJ, 633, 1018

Pei, Y. C. 1992, ApJ, 395, 130

Perley, D. A., Krühler, T., Schulze, S., et al. 2016a, ApJ, 817, 7

Perley, D. A., Tanvir, N. R., Hjorth, J., et al. 2016b, ApJ, 817, 8

Pirard, J. F., Kissler-Patig, M., Moorwood, A., et al. 2004, in Ground-based Instrumentation for Astronomy, eds. A. F. M. Moorwood, \& M. Iye, Proc. SPIE, 5492, 1763

Sánchez-Ramírez, R., Hancock, P. J., Jóhannesson, G., et al. 2017, MNRAS, 464, 4624

Sanders, D. B., Scoville, N. Z., \& Soifer, B. T. 1991, ApJ, 370, 158

Sanders, N. E., Soderberg, A. M., Levesque, E. M., et al. 2012, ApJ, 758, 132

Schady, P., Savaglio, S., Müller, T., et al. 2014, A\&A, 570, A52

Schady, P., Krühler, T., Greiner, J., et al. 2015, A\&A, 579, A126

Schlafly, E. F., \& Finkbeiner, D. P. 2011, ApJ, 737, 103

Scudder, J. M., Ellison, S. L., Torrey, P., Patton, D. R., \& Mendel, J. T. 2012 , MNRAS, 426, 549

Selsing, J., Fynbo, J. P. U., Heintz, K. E., Watson, D., \& Dyrbye, N. 2019, GRB Coordinates Network, 23695, 1

Sirianni, M., Jee, M. J., Benítez, N., et al. 2005, PASP, 117, 1049

Skrutskie, M. F., Cutri, R. M., Stiening, R., et al. 2006, AJ, 131, 1163

Solomon, P. M., Downes, D., Radford, S. J. E., \& Barrett, J. W. 1997, ApJ, 478 144

Spergel, D. N., Verde, L., Peiris, H. V., et al. 2003, ApJS, 148, 175

Stanway, E. R., Levan, A. J., Tanvir, N. R., Wiersema, K., \& van der Laan, T. P. R. 2015, ApJ, 798, L7

Svensson, K. M., Levan, A. J., Tanvir, N. R., Fruchter, A. S., \& Strolger, L. G. 2010, MNRAS, 405, 57

Takahashi, M. 2019, 36th International Cosmic Ray Conference (ICRC2019), 36,606

Tanvir, N. R., Barnard, V. E., Blain, A. W., et al. 2004, MNRAS, 352, 1073

The Fermi GBM team 2019, GRB Coordinates Network, 25551, 1

Tokunaga, A. T., \& Vacca, W. D. 2005, PASP, 117, 421

Vergani, S. D., Salvaterra, R., Japelj, J., et al. 2015, A\&A, 581, A102

Vernet, J., Dekker, H., D'Odorico, S., et al. 2011, A\&A, 536, A105

Violino, G., Ellison, S. L., Sargent, M., et al. 2018, MNRAS, 476, 2591

Wang, X.-Y., He, H.-N., Li, Z., Wu, X.-F., \& Dai, Z.-G. 2010, ApJ, 712, 1232
Wang, W.-H., Chen, H.-W., \& Huang, K.-Y. 2012, ApJ, 761, L32

Whitaker, K. E., Franx, M., Leja, J., et al. 2014, ApJ, 795, 104

Wright, E. L., Eisenhardt, P. R. M., Mainzer, A. K., et al. 2010, AJ, 140, 1868

Wuyts, E., Kurk, J., Förster Schreiber, N. M., et al. 2014, ApJ, 789, L40

Wuyts, E., Wisnioski, E., Fossati, M., et al. 2016, ApJ, 827, 74

Young, J. S., Xie, S., Kenney, J. D. P., \& Rice, W. L. 1989, ApJS, 70, 699

Zahid, H. J., Yates, R. M., Kewley, L. J., \& Kudritzki, R. P. 2013, ApJ, 763, 92

1 Instituto de Astrofísica de Andalucía, Glorieta de la Astronomía s/n, 18008 Granada, Spain e-mail: adeugartepostigo@gmail.com

2 DARK, Niels Bohr Institute, University of Copenhagen, Lyngbyvej 2, 2100 Copenhagen $\varnothing$, Denmark

3 European Southern Observatory, Alonso de Córdova, 3107, Vitacura, Santiago 763-0355, Chile

4 Joint ALMA Observatory, Alonso de Córdova, 3107, Vitacura, Santiago 763-0355, Chile

5 Anton Pannekoek Institute for Astronomy, University of Amsterdam, Science Park 904, 1098 XH Amsterdam, The Netherlands

6 Department of Astrophysics/IMAPP, Radboud University Nijmegen, Nijmegen, The Netherlands

7 Department of Physics, University of Warwick, Coventry CV4 7AL, UK

8 Astronomical Observatory Institute, Faculty of Physics, Adam Mickiewicz University, ul. Słoneczna 36, 60-286 Poznań, Poland

9 The Cosmic Dawn Center (DAWN), Denmark

10 Niels Bohr Institute, University of Copenhagen, Lyngbyvej 2, 2100 Copenhagen $\varnothing$, Denmark

11 Department of Particle Physics and Astrophysics, Weizmann Institute of Science, Rehovot 7610001, Israel

12 GEPI, Observatoire de Paris, PSL University, CNRS, 5 place Jules Janssen, 92190 Meudon, France

13 Institut d'Astrophysique de Paris, Sorbonne Université, CNRS, UMR7095, 75014 Paris, France

14 Department of Physics and Astronomy, University of Leicester, Leicester LE1 7RH, UK

15 INAF - Osservatorio Astronomico di Brera, Via E. Bianchi 46, 23807 Merate, LC, Italy

16 ASI - Space Science Data Centre, Via del Politecnico snc, 00133 Rome, Italy

17 INAF - Osservatorio Astronomico di Roma, Via Frascati 33, 00040 Monte Porzio Catone, RM, Italy

18 Department of Astronomy and Space Sciences, Istanbul University, 34119, Beyazit Istanbul, Turkey

19 Space Telescope Science Institute, 3700 San Martin Drive, Baltimore, MD 21218, USA

20 Department of Physics and Astronomy, Clemson University, Clemson, SC 29634, USA

21 Centre for Astrophysics and Cosmology, Science Institute, University of Iceland, Dunhagi 5, 107 Reykjavík, Iceland

22 Department of Physics, The George Washington University, 725 21st Street NW, Washington, DC 20052, USA

23 Astronomy, Physics, and Statistics Institute of Sciences (APSIS), The George Washington University, Washington, DC 20052, USA

24 GRAPPA Institute, University of Amsterdam, 1098 XH Amsterdam, The Netherlands

25 Astrophysics Research Institute, Liverpool John Moores University, 146 Brownlow Hill, Liverpool L3 5RF, UK

26 INAF - Osservatorio di Astrofisica e Scienza dello Spazio, Via Piero Gobetti 93/3, 40129 Bologna, Italy

27 Department of Astronomy and Astrophysics, Pennsylvania State University, 525 Davey Laboratory, University Park, PA 16802, USA

28 INAF - Istituto di Astrofisica Spaziale e Fisica Cosmica, Via A. Corti 12, 20133 Milano, Italy

29 INAF, Istituto di Astrofisica e Planetologia Spaziali, Via Fosso del Cavaliere 100, 00133 Roma, Italy

30 CAS Key Laboratory of Space Astronomy and Technology, National Astronomical Observatories, Chinese Academy of Sciences, Beijing 100101, PR China 